\title{
ON A SUPERLINEAR ELLIPTIC BOUNDARY VALUE PROBLEM AT RESONANCE
}

\author{
P. J. MCKENNA
}

ABSTRACT. Semilinear partial differential equations of the type $-\Delta u-\lambda_{2} u$ $+e^{u}=h$ are studied and existence and multiplicity results obtained.

1. Introduction. Many recent papers have studied the problem

$$
\begin{aligned}
L u+f(u) & =h(x) \quad \text { in } \Omega, \\
B u & =0 \quad \text { on } \partial \Omega,
\end{aligned}
$$

where $L$ is a nonnegative elliptic operator with kernel and $f$ is a function satisfying $f(+\infty)>f(-\infty)$ without growth restrictions. For example, if $f(u)=e^{u}$ then necessary and sufficient conditions on $h$ are known for equation (1) to have a solution [5]. An obvious next step is to examine the case where $L$ is no longer nonnegative but is only negative on a one-dimensional space. We shall study, with fairly general methods, the problem

$$
\begin{array}{rlrl}
-\Delta u-\lambda_{2} u+e^{u} & =h(x) & \text { in } \Omega, \\
\partial u / \partial n & =0 \quad \text { on } \partial \Omega,
\end{array}
$$

where $\lambda_{2}$ is the first nonzero eigenvalue of $-\Delta$ with Neumann boundary conditions. If instead of $e^{u}$ we had a bounded nonlinearity $f(u)$, then [4] shows that the existence of solutions of (2) is dependent on the projection of $h$ onto the eigenspace corresponding to $\lambda_{2}$. The surprising result here is that the existence or nonexistence of solutions of (2) is dependent also on the projection of $h$ onto the kernel of the Laplacian.

2. A typical example. We shall consider the problem

$$
\begin{aligned}
-\Delta u-\lambda_{2} u+e^{u} & =h_{1}(x)+t \quad \text { in } \Omega, \\
\partial u / \partial n & =0 \quad \text { on } \partial \Omega,
\end{aligned}
$$

where $\int h_{1}=0, h_{1} \in L^{\infty}(\Omega)$. In [5] it was shown that this equation need not have a solution and, for completeness, we include the example here. Rewrite problem (3) as $-\Delta u+f(u)=h_{1}(x)+t$ and observe that inf $f(x)=$ $\inf \left(-\lambda_{2} x+e^{x}\right)=N>-\infty$. Thus if $t<N$, by projection onto the kernel vector 1 , we have $N|\Omega| \leqslant \int f(u)=\int t<N|\Omega|$ which contradicts the existence of a solution $u$. We now prove a complementary result.

Received by the editors September 19, 1977 and, in revised form, July 18, 1978.

AMS (MOS) subject classifications (1970). Primary $35 \mathrm{~J} 60$.

Key words and phrases. Elliptic boundary value problem, monotone nonlinearities. 
THEOREM. Given any $h_{1} \in L^{\infty}, \int h_{1}=0$, there exists a number $\gamma$ so that problem (3) has at least two solutions if $t>\gamma$, at least one solution if $t=\gamma$, and has no solutions if $t<\gamma$.

Proof. First, by an application of the maximum principle, we may conclude that if the number $M$ satisfies $-\lambda_{2} M+e^{M}>\left\|h_{1}\right\|_{\infty}+|t|$, then any solution $u$ of (3) satisfies $u \leqslant M$ almost everywhere.

We now introduce the Nemytsky operator $N$ in $L^{2}$ by $D(N)=\left\{u \in L^{2} \mid u\right.$ is bounded above a.e. in $\Omega\}, N u=e^{u}$ and let $L u=-\Delta u-\lambda_{2} u$. We assume, without loss of generality, that $|\Omega|=1$. Thus, the operator $P$ defined by $P u=\int_{\Omega} u d x$ is the orthogonal projection on the vector 1 .

We now use the alternative method [1] to split the equation

$$
L u+N u=h_{1}+t
$$

into the equivalent system of equations

$$
\begin{aligned}
L u_{1}+(I-P) N\left(c+u_{1}\right) & =h_{1}, \\
-\lambda_{2} c+P N\left(c+u_{1}\right) & =t,
\end{aligned}
$$

where $u_{1} \in(I-P) L^{2}$.

For each real number $c$, let $T_{c}$ be the operator defined by

$$
T_{c} u_{1}=L u_{1}+(I-P) N\left(c+u_{1}\right)
$$

so equation (5a) becomes $T_{c} u_{1}=h_{1}$. We shall first show that this equation is uniquely solvable.

For each $M \geqslant 0$, we introduce the $C^{1}$ cut-off function

$$
g_{M}(x)=\left\{\begin{array}{l}
e^{x}, \quad x \leqslant M, \\
e^{M}(x-M)+e^{M}, \quad x \geqslant M,
\end{array}\right.
$$

and let $N_{M}$ be the associated Nemytsky operator defined on all of $L^{2}$. Corresponding to equation (5a) we have the cut-off equation

$$
L u_{1}+(I-P) N_{M}\left(c+u_{1}\right)=h_{1} .
$$

If we define the operator $T_{c}^{M}$ in $\langle 1\rangle^{\perp}$ by

$$
T_{c}^{M} u_{1}=L u_{1}+(I-P) N_{M}\left(c+u_{1}\right)
$$

then equation $\left(5 \mathrm{a}_{M}\right)$ becomes $T_{c}^{M} u_{1}=h_{1}$. Notice that $N_{M}$ is everywhere defined, bounded and Lipschitzian as a map from $L^{2}(\Omega)$ to $L^{2}(\Omega)$. Furthermore, $N_{M}$ is strictly monotone and thus the map $u_{1} \rightarrow(I-P) N_{M}\left(c+u_{1}\right)$ is monotone and Lipschitzian for fixed $c$ on $(I-P) L^{2}$. Since $L(I-P)$ is maximal monotone, the map $T_{c}^{M}$ is maximal monotone on $(I-P) L^{2}$. We now turn to the task of showing that $T_{c}^{M}$ is coercive on $(I-P) L^{2}$.

First, assuming that $M \geqslant|c|$, a routine calculation shows that

$$
\left(T_{c}^{M} u_{1}, u_{1}\right) \geqslant\left(T_{c}^{|c|} u_{1}, u_{1}\right) \quad \text { for all } u_{1},
$$

and hence, by establishing coercive estimates for $T_{c}^{|c|}$ we obtain coercive estimates for $T_{c}^{M}$ which depend on $c$ but are independent of $M$. We shall use the 
following lemma, whose proof we postpone to the end of the theorem.

LemMa. There exists $\gamma_{0}>0, R_{c}>0$ so that

$$
\frac{\left(T_{c}^{|c|} u_{1}, u_{1}\right)}{\left\|u_{1}\right\|} \geqslant \gamma_{0}\left\|u_{1}\right\|^{1 / 3} \quad \text { for all }\left\|u_{1}\right\| \geqslant R_{c}
$$

where $\gamma_{0}$ is independent of $c$ and $R_{c}$ depends continuously on $c$.

From (6) and (7) it is clear that $\left(5 \mathrm{a}_{M}\right)$ has a unique solution $u_{1}$ satisfying

$$
\left\|u_{1}\right\| \leqslant \max \left\{R_{c},\left\|h_{1}\right\|^{3} / \gamma_{0}^{3}\right\} \text {. }
$$

Since

$$
g_{M}(c+x) \leqslant e^{c}+x g_{M}(x+c)
$$

and

$$
\left(L u_{1}, u_{1}\right)+\int u_{1} g_{M}\left(c+u_{1}\right)=\left(h_{1}, u_{1}\right)
$$

it follows that

$$
\int g_{M}\left(c+u_{1}\right) \leqslant e^{c}+\left\|h_{1}\right\|\left\|u_{1}\right\| \leqslant K_{c}
$$

by (8), where $K_{c}$ depends continuously on $c$ but is independent of $M$. If now the constant $M$ is chosen so that $M \geqslant|c|$ and

$$
-\lambda_{2} M+e^{M}>\left\|h_{1}\right\|_{\infty}+\lambda_{2}|c|+K_{c}
$$

then $u=c+u_{1}$ is a solution of

$$
-\Delta u-\lambda_{2} u+g_{M}(u)=h_{1}+\int_{M} g\left(c+u_{1}\right) d x-\lambda_{2} c
$$

and by the maximum principle and (11) we have $u \leqslant M$. Thus $u_{1}$ is also a solution of equation (5a). This shows that for each real number $c$, equation (5a) has a unique solution $u_{1}=\tau(c)$ and the solution $u_{1}$ is independent on the “cut-off" parameter $M$.

We now show that $N(c+\tau(c))$ is continuously dependent on $c$. Let $\phi_{2}$ span the eigenspace corresponding to the eigenvalue $\lambda_{2}$. Write $\tau(c)=\tau_{1}(c)+$ $\tau_{2}(c)$ where $\tau_{1}(c)=\alpha \phi_{2}$ and $\tau_{2}(c) \perp\left\{1, \phi_{2}\right\}$. Let $c_{n} \rightarrow c_{0}$. It follows from the boundedness of the set $\left\{c_{n}, c_{0}\right\}_{n=1}^{\infty}$ and the estimate (7) that $\left\{\tau\left(c_{n}\right)\right\}$ is bounded in $L^{2}$. First we show that $\tau_{2}(c)$ is a continuous function of $c$. Indeed, for some $\beta$ we have

$$
\begin{aligned}
\beta \| \tau_{2}\left(c_{n}\right) & -\tau_{2}\left(c_{0}\right) \|^{2} \leqslant\left(L\left(\tau_{2}\left(c_{n}\right)-\tau_{2}\left(c_{0}\right)\right), \tau_{2}\left(c_{n}\right)-\tau_{2}\left(c_{0}\right)\right) \\
= & -\left(N\left(c_{n}+\tau\left(c_{n}\right)\right)-N\left(c_{0}+\tau\left(c_{0}\right)\right), \tau_{2}\left(c_{n}\right)-\tau_{2}\left(c_{0}\right)\right) \\
\leqslant & -\left(N\left(c_{n}+\tau\left(c_{n}\right)\right)-N\left(c_{0}+\tau\left(c_{0}\right)\right),\left(c_{n}+\tau\left(c_{n}\right)\right)-\left(c_{0}+\tau\left(c_{0}\right)\right)\right) \\
& +2 \rho\left\|c_{n}-c_{0}\right\| .
\end{aligned}
$$


Here we have used the facts that

$$
\left(N\left(c_{n}+\tau\left(c_{n}\right)\right), \phi_{2}\right)=\left(N\left(c_{0}+\tau\left(c_{0}\right)\right), \phi_{2}\right)=\left(h_{1}, \phi_{2}\right),
$$

so that $\left(N\left(c_{n}+\tau\left(c_{n}\right)\right)-N\left(c_{0}+\tau\left(c_{0}\right)\right), \tau_{1}\left(c_{n}\right)-\tau_{1}\left(c_{0}\right)\right)=0$, and that $\| N\left(c_{n}\right.$ $\left.+\tau\left(c_{n}\right)\right) \| \leqslant \rho$ for some $\rho$. Since the first term on the right is negative and the second term is tending to zero, we conclude that $\tau_{2}\left(c_{n}\right) \rightarrow \tau_{2}\left(c_{0}\right)$.

Since the $\tau_{1}\left(c_{n}\right)$ are bounded, it follows that there exists a convergent subsequence $\tau_{1}\left(c_{n}\right) \rightarrow x$. Now observe that by taking limits, we have

$$
\left(N\left(c_{0}+x+\tau_{2}\left(c_{0}\right)\right)-N\left(c_{0}+\tau_{1}\left(c_{0}\right)+\tau_{2}\left(c_{0}\right)\right), x-\tau_{1}\left(c_{0}\right)\right)=0
$$

However $(N(y)-N(z), y-z)=0$ implies $N(y)=N(z)$, and thus we have that $N\left(c_{0}+x+\tau_{2}\left(c_{0}\right)\right)=N\left(c_{0}+\tau_{1}\left(c_{0}\right)+\tau_{2}\left(c_{0}\right)\right)$. Therefore $N(c+\tau(c))$ is a continuous function of $c$.

Now we must show that for suitable choices of $t$, equation (5b) which takes the form

$$
\Phi(c)=-\lambda_{2} c+\int N(c+\tau(c)) d x=t
$$

is satisfied. Observe that as $c \rightarrow-\infty, \Phi(c) \rightarrow+\infty$. Also observe that by the earlier counterexample $\lim _{c \rightarrow+\infty} \Phi(c)=m>-\infty$. Suppose $m<+\infty$. Then we can find a sequence $c_{n}$ such that $c_{n} \rightarrow+\infty$ and $\Phi\left(c_{n}\right) \rightarrow m$, which contradicts the a priori bound by the maximum principle on solutions $c+\tau(c)$. Thus as $c \rightarrow+\infty, \Phi(c) \rightarrow+\infty$. Taking $\gamma=\min _{c \in \mathbf{R}} \Phi(c)$ we have shown that (12), and thus (3) has at least two solutions if $t>\gamma$, at least one solution if $t=\gamma$ and no solutions if $t<\gamma$. Thus, when we have proved the coercive lemma, the proof is complete.

Proof of Lemma. We shall write $u_{1}=d \phi_{2}+u_{2}$ and establish (7) for two separate cases.

Case $1 .|d|^{2 / 3} \leqslant\left\|u_{2}\right\|$. In this case we have

$$
\begin{gathered}
\left\|u_{1}\right\|^{2}=|d|^{2}+\left\|u_{2}\right\|^{2} \leqslant\left\|u_{2}\right\|^{3}+\left\|u_{2}\right\|^{2} \leqslant 2\left\|u_{2}\right\|^{3}, \\
\left(L u_{1}, u_{1}\right) \geqslant\left(\lambda_{3}-\lambda_{2}\right)\left\|u_{2}\right\|^{2} \geqslant 0, \\
\left(N_{|c|}\left(c+u_{1}\right), u_{1}\right)=\left(N_{|c|}\left(c+u_{1}\right)-N_{|c|}(c), u_{1}\right)+\left(N_{|c|}(c), u_{1}\right) \\
\geqslant 0-\left\|N_{|c|}(c)\right\|\left\|u_{1}\right\| \geqslant-e^{c}\left\|u_{1}\right\|,
\end{gathered}
$$

and hence,

$$
\frac{\left(T_{c}^{|c|} u_{1}, u_{1}\right)}{\left\|u_{1}\right\|} \geqslant \frac{\left(\lambda_{3}-\lambda_{2}\right)}{2^{2 / 3}}\left\|u_{1}\right\|^{1 / 3}-e^{c} \quad \text { for all } u_{1} .
$$


Case 2. $\left\|u_{2}\right\| \leqslant|d|^{2 / 3}$. Here we have

$$
\begin{aligned}
& \left\|u_{1}\right\|^{2} \leqslant|d|^{2}+|d|^{4 / 3} \leqslant 2|d|^{2}, \\
& \left(N_{|c|}\left(c+u_{1}\right), u_{1}\right)=\left(d \phi_{2}, N_{|c|}\left(c+u_{1}\right)\right)+\left(u_{2}, N_{|c|}\left(c+u_{1}\right)\right) \\
& =\left(d \phi_{2}, N_{|c|}\left(d \phi_{2}\right)\right)+\left(d \phi_{2}, N_{|c|}\left(c+u_{1}\right)-N_{|c|}\left(d \phi_{2}\right)\right) \\
& +\left(u_{2}, N_{|c|}(c)\right)+\left(u_{2}, N_{|c|}\left(c+u_{1}\right)-N_{|c|}(c)\right) \\
& \geqslant\left(d \phi_{2}, N_{|c|}\left(d \phi_{2}\right)\right)-d e^{|c|}\left\|c+u_{2}\right\|-\left\|u_{2}\right\| e^{c}-\left\|u_{2}\right\| e^{|c|}\left\|u_{1}\right\| \\
& \geqslant\left(d \phi_{2}, N_{|c|}\left(d \phi_{2}\right)\right)-|d| e^{|c|}|c|-|d| e^{|c|}|d|^{2 / 3}-|d|^{2 / 3} e^{|c|}-|d|^{2 / 3} e^{|c|} \sqrt{2}|d| \\
& \geqslant\left(d \phi_{2}, N_{|c|}\left(d \phi_{2}\right)\right)-(4+|c|) e^{|c|}|d|^{5 / 3} \text {. }
\end{aligned}
$$

To estimate $\left(d \phi_{2}, N_{|c|}\left(d \phi_{2}\right)\right)$, set $\mu=\sup \phi_{2}(x)$ and $\gamma=$ inf $\phi_{2}(x)$. Clearly $-\infty<\gamma<0<\mu<+\infty$. Consider the case when $d>0$ and let

$$
\Omega_{1}=\left\{x \in \Omega \mid \phi_{2}(x) \geqslant \mu / 2\right\} \quad \text { and } \quad \Omega_{2}=\left\{x \in \Omega \mid \phi_{2}(x)<\mu / 2\right\} \text {. }
$$

Note that inf $x g_{M}(x)=-1 / e$ and hence if $d \geqslant 2|c| / \mu$, then $d \phi_{2}(x) \geqslant|c|$ for all $x \in \Omega_{1}$, and

$$
\begin{aligned}
\left(d \phi_{2}, N_{|c|}\left(d \phi_{2}\right)\right) & =\int d \phi_{2} g_{|c|}\left(d \phi_{2}\right)=\int_{\Omega_{1}}+\int_{\Omega_{2}} \\
& \geqslant \int_{\Omega_{1}} d \phi_{2} e^{|c|}\left[d \phi_{2}-|c|+1\right]-\frac{\left|\Omega_{2}\right|}{e} \\
& \geqslant \frac{1}{4} d^{2} e^{|c|} \mu^{2}\left|\Omega_{1}\right|-d e^{|c|}|c|-\frac{1}{e} .
\end{aligned}
$$

A similar argument works for $d<0$. Combining all these inequalities, we conclude that there exists a constant $a>0$, depending only on the eigenvector $\phi_{2}$, such that

$$
\begin{aligned}
\left(N_{|c|}\left(c+u_{1}\right), u_{1}\right) & \geqslant|d|^{2} e^{|c|} a-|d| e^{|c|}|c|-\frac{1}{e}-(4+|c|) e^{|c|} d^{5 / 3} \\
& \geqslant \frac{1}{2} a e^{|c|}\left\|u_{1}\right\|^{2}-(5+2|c|) e^{|c|}\left\|u_{1}\right\|^{5 / 3}
\end{aligned}
$$

and hence,

$$
\frac{\left(T_{c}^{|c|} u_{1}, u_{1}\right)}{\left\|u_{1}\right\|} \geqslant \frac{a}{2}\left\|u_{1}\right\|-(5+2|c|) e^{|c|}\left\|u_{1}\right\|^{1 / 3}
$$

for all $\left\|u_{1}\right\| \geqslant 2 \sqrt{2}|c| / \min \{\mu,-\gamma\}$. The estimate (7) now follows from (13) and (14).

3. Remarks and generalizations. Monotonicity is essential to this method. While equation (5a) may be solved by compactness methods, it is not clear that such methods would yield a set of solutions which depend continuously on c. Apart from this restriction it is clear that these methods apply to any nonlinearity $f(u)$ satisfying $\lim _{u \rightarrow+\infty} f(u) / u>\lambda_{2}$ and $\lim _{u \rightarrow-\infty} f(u)>-\infty$. 
The use of the maximum principle to cut off the nonlinearity occurred in two places. First, by making $N$ Lipschitzian, it ensured that the sum of the two maximal monotone operators in $(5 \mathrm{a})$ was also maximal which was necessary to guarantee that ( $5 \mathrm{a}$ ) had a solution. This equation could also be solved by compactness, using the monotonicity to guarantee continuity of the solution $\tau(c)$.

However, it was also necessary to obtain information on $\lim _{c \rightarrow+\infty} \Phi(c)$ which was necessary for information on the multiplicity of solutions and appears essential here. Without the maximum principle, one could only conclude that for each $h_{1}$ satisfying $\int h_{1}=0$, there exists an open interval $(\gamma, \infty)$ such that equation (3) has at least one solution if $t \in(\gamma, \infty)$ and has no solution if $t \in(-\infty, \gamma)$. This conclusion would apply to higher order problems, for example

$$
\begin{gathered}
\Delta^{2} u-\lambda_{2} u+e^{u}=h_{1}+t \quad \text { in } \Omega, \\
\partial u / \partial n=\partial \Delta / \partial n=0 .
\end{gathered}
$$

The methods used here would apply equally well to the Laplacian with Dirichlet boundary conditions, or any operator whose lowest eigenvector does not change sign.

The problem

$$
\begin{aligned}
-\Delta u-\lambda_{N} u+e^{u}=h & \text { in } \Omega, \\
\partial u / \partial n=0 & \text { on } \partial \Omega
\end{aligned}
$$

appears more difficult. These methods would only yield that for all $h_{1} \perp\left\{\phi_{i}\right\}_{i<N-1}$, then there exists some $h_{2}, h_{2}=\sum_{i=1}^{N-1} c_{i} \phi_{i}$ such that (16) has a solution. This result is surely not precise. Observe that a solution of (2) is also a solution of the Landesman-Lazer problem

$$
\begin{aligned}
-\Delta u-\lambda_{2} u+g_{M}^{*}(u) & =h_{1}+t \quad \text { in } \Omega, \\
\partial u / \partial n & =0 \quad \text { on } \partial \Omega,
\end{aligned}
$$

where $g_{M}^{*}$ is a smooth bounded monotone function satisfying $g_{M}^{*}(M)-\lambda_{2} u>$ $\left\|h_{1}\right\|_{\infty}+|t|$. Consequently, by the work of Nirenberg [4], any solution of this problem is smooth.

The use of monotone operator techniques in the alternative method is not new. The author wishes to acknowledge the inspiration of $\mathrm{L}$. Cesari, from whom he learned this technique. A comprehensive survey may be found in [1].

\section{REFERENCES}

1. L. Cesari, Functional analysis and nonlinear differential equations, Functional Analysis and Nonlinear Differential Equations (Cesari, Kannan, Schuur, Editors), Marcel Dekker, New York, 1976.

2. S. Fucik, Remarks on superlinear boundary value problems, Bull. Austral. Math. Soc. 16 (1977), 181-188. 
3. P. Hess, On strongly nonlinear elliptic problems, Functional Analysis, Lecture Notes in Pure and Appl. Math., vol. 18, Marcel Dekker, New York, 1976.

4. L. Nirenberg, An application of generalized degree to a class of nonlinear problems, Troisième Coll. sur l'Analyse Functionelle, Vander, Louvain, 1971, pp. 57-74.

5. P. J. McKenna and J. Rauch, Strongly nonlinear perturbations of elliptic boundary value problems with kernel, Differential Equations 28 (1978), 253-265.

6. P. J. McKenna, On the reduction of a semilinear hyperbolic problem by monotone operator methods to an alternative problem, Houston J. Math. (to appear).

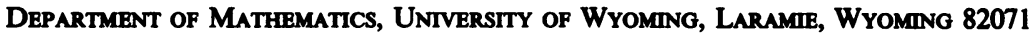

Current address: Department of Mathematics, University of Florida, Gainesville, Florida 32611 